\title{
Lipid-Polymer Membranes as Carriers for L-Tryptophan: Molecular and Metabolic Properties
}

\author{
Fernández Ruocco María Julieta ${ }^{1,2}$, Siri Macarena ${ }^{1,2}$, Igartúa Daniela ${ }^{1,2}$, \\ Prieto María Jimena ${ }^{1,2}$, Alonso Silvia Del Valle ${ }^{1,2}$, Chiaramoni Nadia Silvia ${ }^{1,2^{*}}$ \\ ${ }^{1}$ Laboratory of Biomembranes, Department of Science and Technology, \\ Universidad Nacional de Quilmes, Buenos Aires, Argentina \\ ${ }^{2}$ Instituto Multidisciplinario de Biología Celular, Consejo Nacional de Investigaciones Científicas y Técnicas, \\ Buenos Aires, Argentina \\ Email: "nschiara@unq.edu.ar
}

Received January 1, 2013; revised February 5, 2013; accepted February 16, 2013

\begin{abstract}
Polymerized liposomes encapsulating L-tryptophan were studied with the aim to characterize them as drug delivery systems for the treatment of several metabolic diseases that need an increased systemic L-tryptophan concentration. polymerized liposomes were obtained by UV irradiation of vesicles containing 1,2-bis(10,12-tricosadiynoyl)-sn-glycero-3-phosphocholine (DC8,9PC) and 1,2-dimyristoyl-sn-glycero-3-phosphocholine (DMPC) in a 1:1 molar ratio, in the presence of 10 and $50 \mathrm{~mol} \%$ of L-tryptophan (respect to total lipid concentration). Polymerization efficiency was studied spectrophotometrically. Also, bilayer packing at the polar head region was followed with the Merocyanine 540 (MC540) and specific interactions in the lipopolymers were studied by FTIR. High L-tryptophan concentrations (50 mol\% respect to total lipid concentration) induced a higher amount of six- and nine-unit polymers. This phenomenon was induced because the L-tryptophan located outside the lipid membrane was included in it during the polymerization process and was thus responsible for the better accommodate of the polar head region. This was not possible with the lower amount of L-tryptophan (10 mol\%). The stability of lipopolymers with different amounts of L-tryptophan was studied through release profiles. Polymerized liposomes with $50 \mathrm{~mol} \%$ of L-tryptophan were able to retain around $80 \%$ of the amino acid after 24 hours, whereas those with $10 \mathrm{~mol} \%$ of the amino acid were able to retain $20 \%$. The metabolic activity of the Caco-2 cell line was also studied. Cytotoxic effects were low in the presence of polymerized liposomes, rendering a maximum percentage of cell death of $30 \%$. In summary, this work stresses the relevance of nonspecific drug-polymerized membrane binding on L-tryptophan pharmacological interaction with possible pharmaceutical applications in liposomal drug delivery. Moreover, the absence of significant cytotoxic effects allows the system proposed to be applied in human health.
\end{abstract}

Keywords: Lipopolymers; L-Tryptophan; Drug Delivery

\section{Introduction}

Liposomes, which are useful drug delivery systems, have been used to deliver not only small molecules [1] but also macromolecules such as proteins [2] and DNA [3]. polymerized liposomes can be obtained by a photopolymerization process. Since photo-polymerizable lipids combine the plasticity of lipids with the robustness of polymers, they have received much attention in several biological areas [4]. Research of lipopolymers as tools to be applied in biomedical science started in the early 1980s [5]. Design of lipopolymers relies on two advantages: lipid self-assembly properties and photoactivable bonds in photopolymerizable lipids [6]. Lipopolymers have many potential applications in medicine since they

\footnotetext{
${ }^{*}$ Corresponding author.
}

have a great stability. Polymerizable lipids contain a diacetylene moiety along their acyl chain. These lipids can be polymerized by UV irradiation to form chains of covalently linked lipids in the bilayer. Closely packed and properly ordered, diacetylene lipids undergo polymerization via 1,4-addition to form alternating ene-iyne polymer chains upon irradiation with $254 \mathrm{~nm}$ light [7]. Lipopolymers can aggregate into a variety of vesicular and non-vesicular assemblies depending on the lipid concentration, structure and processing conditions [8].

L-tryptophan, an essential amino acid, is a vital constituent of proteins and indispensable in human nutrition for maintaining a positive nitrogen balance [9]. It has an amphiphilic character due to the hydrophobic indole ring structure and the hydrophilic amino acid part [10]. Indole, the aromatic side chain element of L-tryptophan, seems 
to play many biological roles, as an anchor or organizing element or as a hydrogen bond donor [11]. L-tryptophan is also a serotonin precursor and can thus affect behavioral patterns such as food consumption and sleeping.

Regarding L-tryptophan interaction with liposomes, when this amino acid is in a membrane protein, it displays strong preference for the lipid membrane interface and is very important for anchoring proteins at the proper longitudinal level. Indole's prefer to orient themselves with their nitrogen pointing towards the hydrophilic part of the membrane. The long-axis of indole's points preferentially in the direction of the membrane plane as depicted in Figure 1 [12,13].

According to Popova A. and Hincha D. (2004), Ltryptophan destabilizes both biological and model membranes during freezing. The degree of destabilization of L-tryptophan, especially at low L-tryptophan concentrations, depends on the lipid composition of the membrane. Membranes made up entirely of phosphatidylcholine or with a mixture of phosphatidylcholine and phosphatidylethanolamine are very strongly affected, while much less destabilization is observed for galactolipid-containing liposomes [14].

The initial observation that physiological changes in tryptophan availability after food consumption could affect serotonin synthesis derives from studies in rats performed in 1971 [15]. However, direct evidence suggesting that variations in tryptophan concentrations affect serotonin release was not obtained until 1987, when it was reported that the rate at which serotonin is released from hypothalamic nerve terminals is coupled to brain tryptophan levels. Accelerations in hypothalamic serotonin synthesis, caused by increasing brain tryptophan levels, result in proportionate increases in the rates of serotonin release during rest with membrane depolarization [16].

Also, since L-tryptophan is a serotonin precursor, it has an anorectic effect and is highly related to anxiety control, stress and insomnia [17]. Brain serotonin appears to be an excellent target for designing drugs to treat obesity. The special importance of serotoninergic neurons in feeding behavior derives in part from the fact that eating

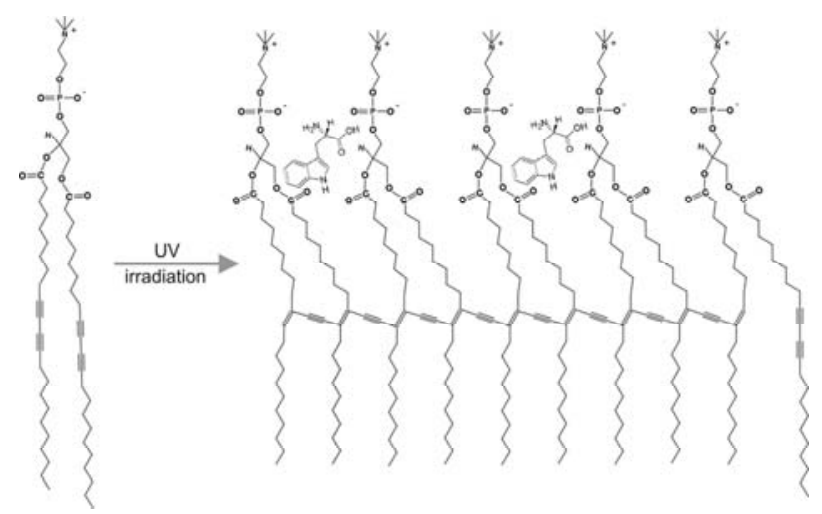

Figure 1. Scheme of polymerization reaction. per se affects the production and release of serotonin. This is because dietary carbohydrates, acting via insulin, decrease the plasma concentrations of large neutral amino acids and increase the uptake of tryptophan in the brain. This, in turn, increases the substrate saturation of tryptophan hydroxylase, the key enzyme that converts this amino acid to serotonin. Paradoxically, dietary proteins - which, unlike carbohydrates or fats, do contain tryptophan-decrease tryptophan's uptake in the brain (because they cause disproportionately great increases in blood levels of other large neutral amino acids, which compete with tryptophan for brain uptake), and thus fail to increase brain tryptophan or serotonin levels [18]. So, the increase in the systemic concentration of pure Ltryptophan could be considered as a possible therapy to treat a problem such as obesity.

Thus, obtaining a liposome system that could deliver L-tryptophan and increase the concentration gradient after oral inoculation will probably lead to an alternative therapy to treat obesity from a behavioral point of view. To achieve this, goal it is first necessary to study and characterize the lipid vehicles from a biophysical and a biochemical point of view. Therefore, the main goal of this work was to obtain polymerized liposomes that can encapsulate L-tryptophan and study some relevant parameters such as polymerization efficiency, structure, release of L-tryptophan and influence on cell metabolism.

\section{Materials and Methods}

\subsection{Materials}

Lipids were purchased from Avanti Polar Lipids (Alabama, USA) and used without further purification. Reagents used in cell culture were purchased from GIBCO (USA). All solvents were of analytical grade or higher.

\subsection{Liposome and Polymer Preparation}

Lipids (6.4 $\mu \mathrm{mol}, 5.2 \mathrm{mg}$ ) were dissolved in chloroform; the solvent was flash-evaporated and left under vacuum for $30 \mathrm{~min}$ [19]. Large multilamellar vesicles (LMVs) were obtained by adding $2 \mathrm{~mL}$ of phosphate buffered saline (PBS) pH 7.4 (with or without L-tryptophan). UV irradiation was accomplished with a Stratalinker 1800 to cross-link and polymerize the diacetylenic LMVs. Polymerization was performed as previously described $[3,8$, 20-23]. Briefly, polymerization cycles were of 360,000 $\mathrm{mJ} / \mathrm{cm} 2$, each of $1650 \mathrm{~s}$ (15 cycles in total), at a wavelength of $254 \mathrm{~nm}$. Temperature was kept at $4^{\circ} \mathrm{C}$ in between cycles. LMVs concentration was kept at $2 \mathrm{mg} / \mathrm{ml}$. The spectra were recorded at different polymerization cycles, in particular at $0,1,5,10$ and 15 . Visible spectra were recorded on a Shimadzu 160-A double beam spectrophotometer, between 400 and $600 \mathrm{~nm}$ for both polym- 
erized and non-polymerized liposomes.

\subsection{L-Tryptophan Incorporation}

L-tryptophan was dissolved in PBS and incorporated in the dry lipid film. Two concentrations were tested: 10 and $50 \mathrm{~mol} \%$ relative to total lipid concentration.

\subsection{In Vitro Release of L-Tryptophan}

The L-tryptophan releasing profile of polymerized liposomes was studied in PBS. Non-encapsulated L-tryptophan was separated by centrifugation [20]. Polymerized liposomes with 10 and $50 \mathrm{~mol} \%$ of L-tryptophan were prepared as described above and $0.2-\mathrm{mL}$ aliquots were withdrawn at different times. The lipid suspension was incubated for $24 \mathrm{~h}$ at $37^{\circ} \mathrm{C}$ under constant agitation. After each sample withdrawing, an aliquot of PBS was added to maintain the original concentration gradient. L-tryptophan was spectrophotometrically quantified from the supernatant by using a UV-visible spectrophotometer Nanodrop 1000 (Thermoscientific, USA). Absorbance was measured at $280 \mathrm{~nm}$.

The release percentage was determined by the following equation:

$$
\mathrm{TL}(\%)=((\mathrm{TLt}+\mathrm{TLt}-1) \times 100) / \mathrm{TT}
$$

where TLt is the concentration of L-tryptophan released at different times, TLt- 1 is the concentration of L-tryptophan released in the previous sample (this was added to avoid error of the concentration gradient) and TT is the total L-tryptophan concentration added to polymerized liposomes. An aliquot was withdrawn immediately after preparation and it was used to calculate the $\%$ of encapsulation.

\subsection{Surface Modifications}

To determine the spectral characteristics of the membrane/solution interface, the visible spectra of the probe merocyanine 540 (MC540) was recorded between 400 and $600 \mathrm{~nm}$ at $10^{\circ} \mathrm{C}$ to maintain the gel phase because this is the structure needed for the polymerization reaction. The MC540 probe is sensitive to the polar environment. In water, the spectra show two maxima (a dimer at $500 \mathrm{~nm}$ and a monomer at $530 \mathrm{~nm}$ ). In a hydrophobic environment, the maximum is shifted towards $530 \mathrm{~nm}$ (MC540 dimer) and $570 \mathrm{~nm}$ (MC540 monomer). The first two are the characteristic absorbance maxima when the membrane is in the gel phase, whereas the second two are the characteristic absorbance maxima when the membrane is in liquid crystalline [24]. The ratio of the absorbance at $570 \mathrm{~nm}$ with respect to $500 \mathrm{~nm}$, called hydrophobicity factor (HF) [22], determines the degree of hydrophobic sites exposed to the interface. The MC540 stock concentration was approximately $1 \mathrm{mg} / \mathrm{ml}$. MC540, freshly prepared, was added in aliquots to give a final concentration of $10-6 \mathrm{M}$. The lipid/ probe ratio was kept at (160:1). The sample was allowed to equilibrate for 2 min at $15^{\circ} \mathrm{C}$ to keep lipids in gel phase, and then measured. To avoid scattering, LMVs without MC540 were used as reference. The lipid concentration was kept at 2 $\mathrm{mg} / \mathrm{ml}$ for the polymerization process and $0.6 \mathrm{mg} / \mathrm{ml}$ for the measurements of the visible spectra. HF was measured in samples with $0,1,5,10$ and 15 polymerization cycles.

\subsection{FTIR Spectroscopy}

Fourier-transform infrared (FTIR) spectroscopy was used to study the interaction between lipopolymers and Ltryptophan as well as the changes induced by the polymerization process. To this end, $0.4 \mathrm{~mL}$ of the liposomal suspension with or without L-tryptophan or polymerization were pipetted onto ATR cells and dried with a hair drier. Infrared spectra were recorded from 3000 to 1000 $\mathrm{cm}^{-1}$ in an IRAffinity-1 Fourier Transform Infrared spectrometer (Shimadzu). Data were analyzed, normalized and baseline corrected with the IRsolution software (Shimadzu). Frequencies were determined by the peak identification routine of the software.

\subsection{Cell Metabolism Activation and Cytotoxicity}

To study the influence of the delivery systems proposed on a cell culture and analyze if there is any cytotoxic effect, the Caco- 2 cell line, a human cell line derived from a colon adeno carcinoma [25], was selected. This cell line, which is widely used in assays that involve drug absorption related to an oral route, was selected because its characteristics are similar to those of the absorptive intestinal epithelium [26]. Several concentrations of polymerized liposomes with 10 and $50 \mathrm{~mol} \%$ of L-tryptophan (relative to total lipid concentration) were seeded in confluent cell culture. Briefly, Caco- 2 cells were seeded in a 96-well plate. Cells were grown in minimum essential medium (MEM) enriched with $15 \% \mathrm{v} / \mathrm{v}$ of bovine fetal serum (BFS), $10 \mathrm{mM}$ sodium pyruvate and a combination of antibiotics and antimycotics. All reagents were purchased from GIBCO (USA). Cells were allowed to grow at $37^{\circ} \mathrm{C}$ in a $5 \% \mathrm{CO}_{2}$ atmosphere. Upon cell confluence, liposomes were seeded in maintenance medium (without BFS). After 4 or 24 hours, the medium was washed and an MTT assay was performed. Cell viability, which depends on metabolic processes, especially mitochondrial respiratory chain, can be evaluated by studying mitochondrial dehydrogenases. MTT is a colorimetric reagent, which turns yellow when oxidized and purple after reduction by mitochondrial enzymes. Absorbance at $570 \mathrm{~nm}$ is a quantitative analysis used to detect reduced 
MTT [27]. The MTT assay was performed in Caco-2 cells treated with polymerized liposomes with 10 and 50 mol\% of L-tryptophan. The lipid concentration was kept at $1 \mathrm{mg} / \mathrm{ml}$. Reduced MTT by cells treated with polymerized liposomes or L-tryptophan was also measured. Cells without liposomes or L-tryptophan were used as controls.

\section{Results and Discussion}

Understanding the structure and function of lipid membranes at the interface is a major challenge in the field of biophysics and requires a combination of structural and functional methods.

L-tryptophan is a serotonin precursor, has an anorectic effect and is highly related to anxiety control, stress and insomnia [16], all of which are extremely important factors in human nutrition. Even more, if the L-tryptophan is carried by an adequate oral delivery vehicle, like DMPC: DC8, 9PC 1:1 mol ratio $[3,8,14,19,20,27]$. Polymerization of diacetylenic phospholipids implies the formation of intra- and intercatenary bonds. Polymers so obtained are highly colored due to their highly conjugated structures [28]. The polymerization degree is determined by absorbance spectra, while the position of these peaks is related to the number of polymeryzed units that are effectively conjugated. The number of these polymerized units can be calculated as previously described [29]. For diacetylenic polymers mixed with DMPC as saturated lipid at a 1:1 lipid molar ratio, there are two populations: one with six polymeric units (maximum at $480 \mathrm{~nm}$ ) and another one with nine polymerized units (maximum at $520 \mathrm{~nm})[14]$.

\subsection{Polymerization Degree}

To study the influence of L-tryptophan on polymer formation, spectra of samples with and without L-tryptophan were recorded after $0,1,5,10$ and 15 cycles of polymerization. Figure 2 depicts absorbance at $480 \mathrm{~nm}$ (a) and $520 \mathrm{~nm}$ (b) as a function of polymerization cycles.

The data obtained showed that samples with high concentration of L-tryptophan polymerized more efficiently than those with low concentration. In the case of the six-unit polymers (Figure 2(a)), $10 \mathrm{~mol} \%$ of L-tryptophan induced a better polymerization but only at the beginning of the process; after 15 cycles, the concentration of six-unit polymers with $10 \mathrm{~mol} \%$ was the same as that without L-tryptophan addition. Regarding nine-unit polymers (Figure 2(b)), $10 \mathrm{~mol} \%$ of L-tryptophan had no influence in the polymerization efficiency. A concentration of $50 \mathrm{~mol} \%$ of L-tryptophan induced a better polymerization in both six- and nine-unit polymers.

A more efficient polymerization may be achieved when the more ordered acyl lipid chains are aligned

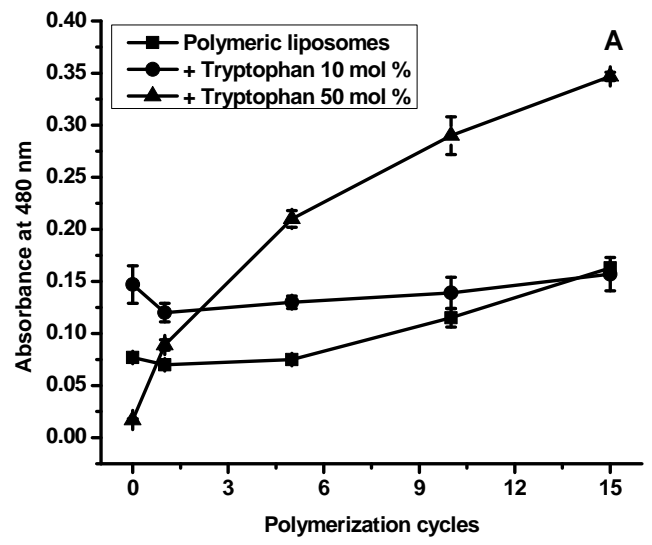

(a)

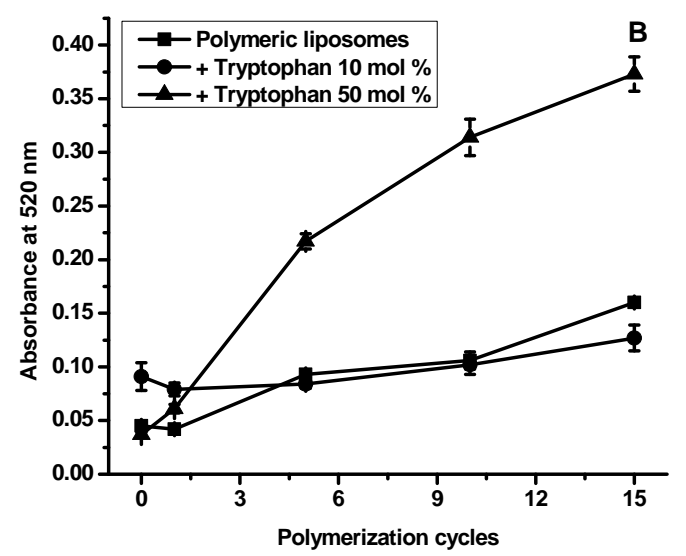

(b)

Figure 2. Polymerization degree. Figure 1 shows absorbance at $480 \mathrm{~nm}$ (a) corresponding to six polymerized units effectively conjugated and at $520 \mathrm{~nm}$ (b) corresponding to nine polymerized units effectively conjugated as a function of polymerization cycles. Data are presented as mean \pm SD of four independent experiments.

closer to each other, improving the chemical topotactic reaction.

Several authors have reported that L-tryptophan can interact with the lipid interphase [13]. A high concentration of L-tryptophan can induce a better polymerization during the process when the amino acid keeps entering the interface from the aqueous medium. This is possible because in between UV cycles, samples are incubated at $4^{\circ} \mathrm{C}$, so lipid membranes can be reorganized and L-tryptophan in solution can have access to the interface. A concentration of $10 \mathrm{~mol} \%$ respect to total lipid is not enough to produce this effect, and polymerization is not different from controls.

To gain insights into the polymerization process in relation with liposome structure, particularly surface packing, the MC540 probe was used and HF was determined. Results are shown in Figure 3.

With high L-tryptophan concentrations $(50 \mathrm{~mol} \%$ relative to total lipid concentration), HF decreased. With 


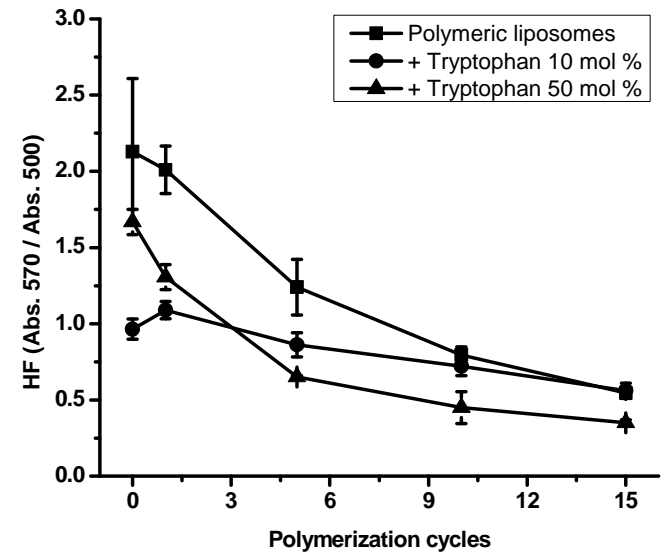

Figure 3. Hydrophobicity factor (HF). Figure 2 shows the HF (Absorbance at $570 \mathrm{~nm} /$ Absorbance at $500 \mathrm{~nm}$ ) as a function of the polymerization cycles. Data are presented as mean \pm SD of four independent experiments.

10 mol\% of L-tryptophan, surface packing showed no changes in comparison with liposomes without L-tryptophan. All preparations were subjected to 15 polymerization cycles. In a previous work [14], we found that only a maximum of $20 \%$ of the monomers are polymerized at the end of the polymerization process and that no further polymerization can be achieved by continuous irradiation of the DC8, 9PC:DMPC mixture [8,30]. The binary system DC8, 9PC:DMPC should be considered as a ternary one after UV irradiation, conformed by DMPC molecules and monomeric and polymerized units of DC8, 9PC. DC8, 9PC polymers represent small segregated domains that can be found in DMPC and DC8, 9PC-rich regions [14].

It has been reported that L-tryptophan has a specific orientation in model lipid membranes [13,31]. L-tryptophan displays strong preference for the lipid membrane interface; in particular, indole prefers to orient with its nitrogen pointing towards the hydrophilic part of the membrane and its long-axis in the direction of the membrane plane [13]. This specific location could be responsible for the increase in surface packing. Regarding the data obtained, the higher L-tryptophan concentration induced higher packing and polymerization efficiency. It is probable that L-tryptophan prefers DMPC-enriched domains rather than $\mathrm{DC} 8,9 \mathrm{PC}$, resulting in ordered diacetylenic domains and improving polymerization efficiency. One of the facts that contributes to this hypothesis is that these domains are more disordered than DC8, 9PC domains, according to their gel-fluid transition-temperature: being DMPC main transition temperature is $23^{\circ} \mathrm{C}$, while that of $\mathrm{DC} 8,9 \mathrm{PC}$ is $45^{\circ} \mathrm{C}$. In DMPC disordered domains, there are more water molecules interacting with the head groups, and, being L-tryptophan a water-soluble amino acid, it should be preferentially at the interface.

\subsection{In Vitro Release}

Encapsulation efficiency of L-tryptophan in the case of polymerized liposomes with $10 \mathrm{~mol} \%$ of the amino acid was $19.81 \pm 1.87$ and in the case of $50 \mathrm{~mol} \%$ it was $17.11 \pm 1.89$. Although percentages are similar greater amount of amino acid was encapsulated in the case of incubation with a higher concentration of L-tryptophan. Releasing profiles are shown in Figure 4.

Data corresponding to percentage of L-tryptophan release are shown in Figure 4. Polymerized liposomes prepared in presence of $50 \mathrm{~mol} \%$ of L-tryptophan released less amino acid than in the case of polymerized liposomes prepared in presence of $10 \mathrm{~mol} \%$ of L-tryptophan. Even though, both curves would be consider as an efficient controlled release system, $50 \mathrm{~mol} \%$ of L-trp has 5 times concentration compared with $10 \mathrm{~mol} \%$. Apparently, high concentration of amino acid not only is useful for pharmacodynamics purposes, it can also stabilize lipopolymers. Polymerized liposomes were efficient in retaining the amino acid probably because of their high rigidity. L-tryptophan was probably in the hydrophilic region and in the aqueous spaces placed between lipid bilayers. The formulations used were multilamellar to increase amino acid encapsulation efficiency.

\subsection{FTIR Spectroscopy}

To corroborate the position of the amino acid in contact with lipid groups, the FTIR spectra of polymerized and non-polymerized liposomes were recorded to identify different interactions that could appear due to the polymerization reaction. Spectra with the vibrational modes are shown in Figure 5.

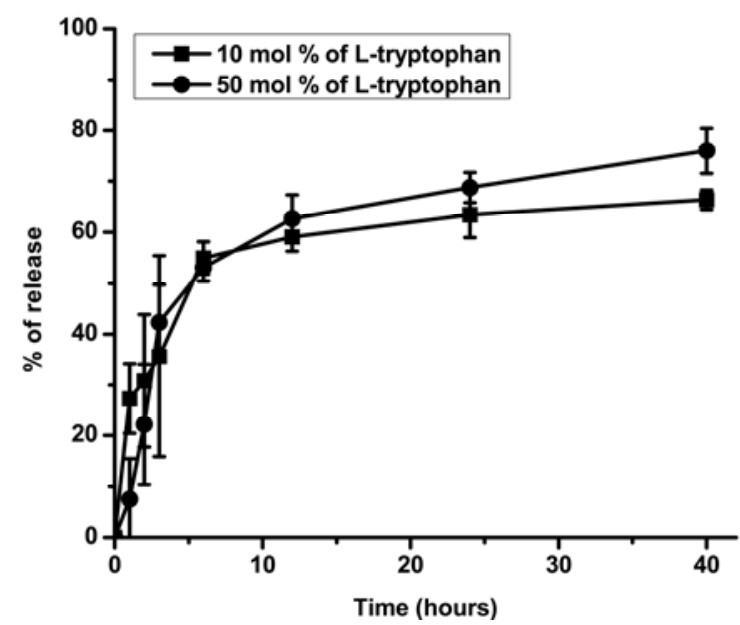

Figure 4. In vitro L-tryptophan release. Figure 3 shows the percentage of L-tryptophan released at different times, calculated as mentioned above. Data are presented as mean \pm SD of three independent experiments. "Significant differences respect to samples with $50 \mathrm{~mol} \%$ of L-tryptophan after performing Tukey's test $(p<0.05)$. 

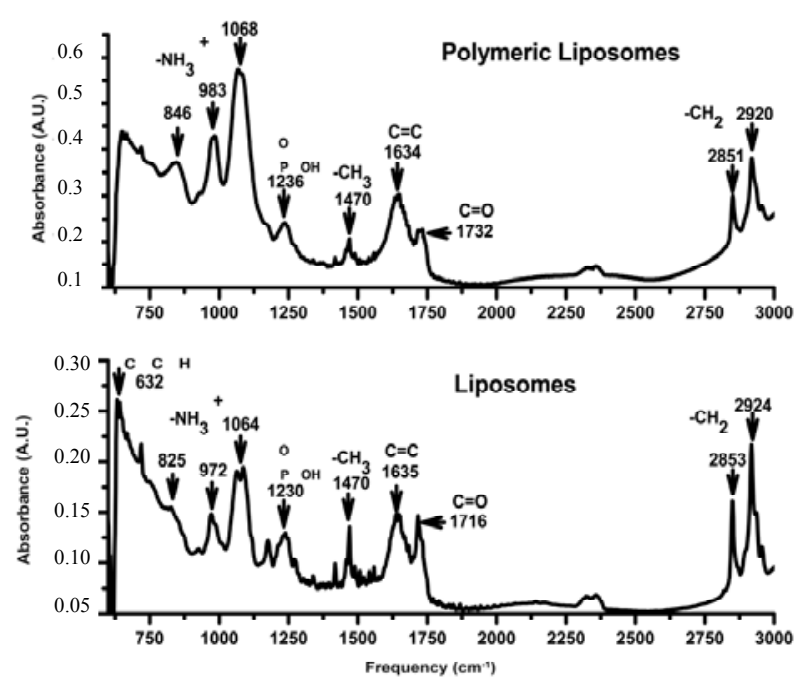

Figure 5. FTIR spectra of DC8, 9PC:DMPC liposomes and polymerized liposomes (top spectrum).

The vibrational modes analyzed were reported by other authors [32]. Methylene symmetric stretching mode $\left(2850 \mathrm{~cm}^{-1}\right)$ and antisymmetric stretching mode $(2920$ $\mathrm{cm}^{-1}$ ) are associated with changes in conformational order [33].

Between 2800 and $3000 \mathrm{~cm}^{-1}$, the spectra showed two peaks: 2850 and $2920 \mathrm{~cm}^{-1}$, corresponding to methylene symmetric and antisymmetric stretching respectively. A decrease in the wavenumber of these bands is associated with an increase in the conformational order of the aliphatic chains of the lipid, resulting from an increase in the number of trans conformers with respect to gauche conformers [33]. The polymerization process induced a decrease in these bands, demonstrating that lipopolymers have increased packing with respect to non-polymerized formulations.

I Non-polymerized liposomes, have the $-\mathrm{C}=\mathrm{O}$ stretching band shifted towards smaller frequencies, which suggests higher hydration in the zone of the polar head near the beginning of the hydrophobic region [34]. This result is in accordance with those obtained in the determination of the HF, where non-polymerized samples exhibited a higher number of surface defects (i.e. higher HF). Also, the hydrogen bonding established with water molecules in non-polymerized liposomes was higher, as the $-\mathrm{C}=0$ stretching band is shifted towards smaller frequencies.

In alkynes, the carbon stretch $\left(632 \mathrm{~cm}^{-1}\right)$ is missing in polymerized liposomes because the triple bond reacts to form double bonds. The absence of this band in polymerized liposomes has also been reported by other authors [32].

FTIR spectra were also recorded in polymerized liposomes with and without L-tryptophan. Absorbance data are shown in Table 1.
Table 1. Peak positions in polymerized liposomes with and without L-tryptophan.

\begin{tabular}{ccc}
\hline Vibrational mode & Polymeric liposomes & $\begin{array}{c}\text { Polymeric liposomes } \\
\text { with L-tryptophan }\end{array}$ \\
\hline$-\mathrm{CH}_{2}$ & 28512920 & 28502920 \\
$-\mathrm{C}=\mathrm{O}$ & 1732 & 1732 \\
$-\mathrm{C}=\mathrm{C}-$ & 1634 & 1635 \\
$-\mathrm{CH}_{3}$ & 1470 & 1467 \\
$-\mathrm{POOH}$ & 1236 & 1236 \\
$-\mathrm{NH}_{3}{ }^{+}$ & 8469831068 & 8589721070 \\
\hline
\end{tabular}

In the case of $\mathrm{POOH}$ vibrational modes, the position of the peak is sensitive to the formation of H-bonds or coulombic interactions, shifting to lower frequencies with increasing bonding [35]. Non-polymerized liposomes exhibited more H-bonding in $\mathrm{POOH}$ vibrational mode than polymerized ones (Figure 3). This is probably because when lipids are closer to each other, like in the case of polymerized formulations, the charges of phosphate groups are probably being repelled and bonding avoided. The presence of L-tryptophan had no influence on -POOH vibrational mode (Table 1), which suggests that this amino acid interacts with the interphase through other groups, but not through - $\mathrm{POOH}$.

Also, L-tryptophan had no influence on $-\mathrm{C}=\mathrm{O}$ or $-\mathrm{CH} 2$ vibrational modes, but changed the vibrational mode of -CH3 $\left(1470 \mathrm{~cm}^{-1}\right)$. In this way, the L-tryptophan that interacts at the interface prolongs its influence up to the end of the acyl chain. This, L-tryptophan may induce chain interdigitation: interacting at the surface of the bilayer, bringing apart some of the lipid headgroup molecules, modiyfng the environment from the surface down into the hydrophobic core, hence changing the vibrational mode of the - $\mathrm{CH} 3$ groups, causing lipid interdigitation $[36,37,38]$.

In the case of $-\mathrm{NH} 3+$ vibrational modes, several changes were induced by the polymerization process and by differential amino acid content. Polymerization induced the $980 \mathrm{~cm}^{-1}$ band shifts toward higher frequencies; this band corresponds to amine skeleton vibration [39] and a higher frequency reflects minor mobility, so $-\mathrm{NH} 3+$ has less freedom degree as a consequence of more rigid surface in polymerized liposomes than in non-polymerized ones. This result is along with those of HF data: polymerized liposomes showed lower HF than non-polymerized ones, which means that polymerized liposomes are more rigid at the interphase than non-polymerized ones. When L-tryptophan is present, the amines skeleton acquires more movement because the amino acid interacts with phosphatidylcholine head groups through the amines, decreasing the amine phospholipid group degrees of freedom. 
This interaction is so strong that it prevents L-tryptophan from being released from the lipopolymer, as reflected in the release assay; only around $20 \%$ of the amino acid is released.

Also, in polymerized liposomes with L-tryptophan there are no modifications in the order of the acyl chains respect to polymerized samples without the amino acid (2850 and $2920 \mathrm{~cm}^{-1}$ bands, Table 1). This could be because, as previously described, polymerized DC8, 9PC is only $20 \%$ of the total diacetylenic lipid [8] and this low percentage did not induced changes in FTIR spectra.

\subsection{Cell Metabolism Activation}

To study if the polymerized liposomes containing Ltryptophan influence metabolism and can induce cytotoxic effects, an MTT assay was performed in Caco-2 cells. The liposome concentration in all assays was 1 $\mathrm{mg} / \mathrm{ml}$. Untreated cells were used as controls. Liposomes and L-tryptophan without liposomes were also tested. Results are shown in Figure 6.

Polymerized liposomes can induce a mild metabolic activation, which is maintained after 24 hours. This has also been reported for other cell lines in the case of polymerized liposomes [24].

When L-tryptophan concentration is $10 \mathrm{~mol} \%$ respects to total lipid concentration, a high metabolic activation is induced in the absence and presence of polymerized liposomes after 4 hours treatment. . Prolonging the treatment for 24 hours led to a large effect: $50 \%$ of the cell culture showed no metabolic activity in the presence of free L-tryptophan. However, when the tryptophan was encapsulated in polymerized liposomes, the cytotoxic effects were lower- only around $15 \%$ of the cell cul-

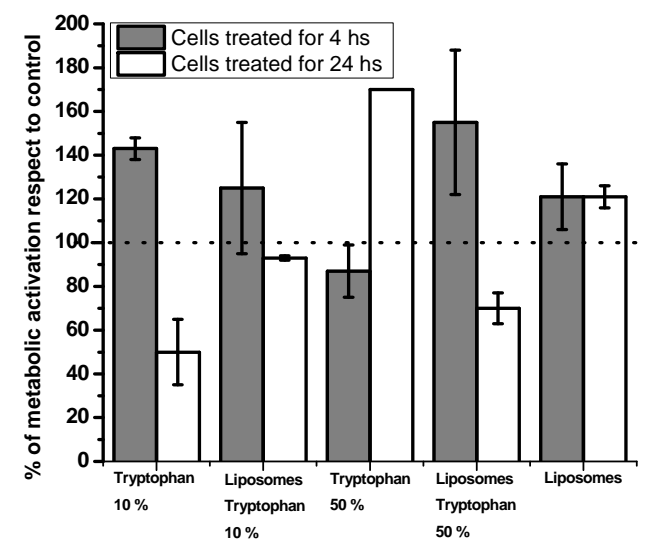

Figure 6. Cell metabolism activation. Figure 6 shows metabolic activation of cells treated with polymerized liposomes and different L-tryptophan concentrations. Untreated cells were taken as a control. Data are presented as mean \pm SD of three independent experiments. The dotted line indicates metabolic activation higher than $100 \%$ respect to the control. ture had no metabolic activity.

The indole side chain of tryptophan is a nitrogen-containing heterocycle, a hydrogen bond donor and an electron-rich aromatic system [13]. Since cell metabolism was measured by the activity of mitochondrial reductases, one hypothesis is that since tryptophan is an electron-rich amino acid, it can induce higher reductase activity after 4 hours because of its oxidative activity. When tryptophan was encapsulated in polymerized liposomes, reductase activity effect after 24 hours increased, probably as a result of the amino acid protection by polymerized liposomes.

In the case of L-tryptophan $50 \%$, metabolic activation remained unmodified after 4 hours but increased when the amino acid was encapsulated in polymerized liposomes. This is probably because of a combined effect: metabolic activation of polymerized liposomes and high L-tryptophan concentration. As mentioned before, polymerization in the presence of high L-tryptophan concentration is more efficient, leading to a more concentrated polymer, and this polymer could have more influence on metabolic activity than the one generated with 10 $\mathrm{mol} \%$ of L-tryptophan.

After 24 hours, cells treated with high L-tryptophan concentrations presented high metabolic activity, while when tryptophan was encapsulated in polymerized liposomes, around $70 \%$ of the cell culture had reductase activity. This effect was probably because of the degradation pathway of the L-tryptophan. Indoleamine 2,3-dioxygenase (IDO) is a heme-containing dioxygenase which catalyzes the first and rate-limiting step in the main pathway of L-tryptophan catabolism in mammals. Much attention has been recently paid to dioxygenase because this metabolic pathway is involved in a variety of physiological functions [40]. Tryptophan catabolism through the IDO pathway is probably involved in the production of antioxidant molecules [41]. These antioxidant molecules could induce a decrease in the antioxidant activity of the mitochondrial dehydrogenases, which is measured with the MTT assay. This effect was observed only in the case of $50 \mathrm{~mol} \%$ of L-tryptophan respect to total lipid concentration probably because of the high amino acid concentration.

Overall, polymerized liposomes did not induce high cytotoxic effects in Caco-2 cells, which makes them secure to apply as vehicles for molecules such as L-tryptophan.

\section{Conclusions}

Polymerized liposomes proposed as amino acid vehicles showed to be able to encapsulate high concentrations of L-tryptophan. This amino acid also induced higher polymerization efficiency, as a result of an increase in packing of the polar region area. 
Polymerized liposomes were also able to retain around $80 \%$ of the L-tryptophan added, even when L-tryptophan concentration was $50 \mathrm{~mol} \%$ relative to total lipid.

When polymerized liposomes are in contact with cells belonging to absorbent epithelia, they do not modify the metabolic activity significantly, resulting in a secure drug delivery system that could be applied to improve the pharmacodynamics of L-tryptophan.

\section{Acknowledgements}

This research was supported by a grants from Universidad Nacional de Quilmes, MINCyT, CONICET and CIC. Silvia del Valle Alonso and Nadia S. Chiaramoni are members of Consejo Nacional de Investigaciones Científicas y Técnicas (IMBICE-CONICET, Argentina). M.J. Prieto from FAN and M. Julieta Fernandez Ruocco and Macarena Siri have fellowships from UNQ and CIC, respectively.

\section{REFERENCES}

[1] B. C. Keller, "Liposomes in Nutrition," Trends in Food Science \& Technology, Vol. 12, No. 1, 2001, pp. 25-31.

[2] N. Hirota, S. Duzgunes and N. Duzgunes, "PhysicoChemical Approach to Targeting Phenomena," Current Drug Discovery Technologies, Vol. 8, No. 4, 2011. doi:10.2174/157016311798109399

[3] N. S. Chiaramoni, J. Gasparri, L. Speroni, M. C. Taira and S. del V. Alonso, "Biodistribution of Liposome/DNA Systems after Subcutaneous and Intraperitoneal Inoculation," Journal of Liposome Research, Vol. 20, No. 3, 2010, pp. 191-201. doi:10.3109/08982100903244518

[4] B. Yavlovich, A. Smith, R. Gupta, K. Blumenthal, A. Blumenthal and A. Puri, "Light-Sensitive Lipid-Based Nanoparticles for Drug Delivery: Design Principles and Future Considerations for Biological Applications," Molecular Membrane Biology, Vol. 25, No. 7, 2010, pp. 364-381. doi:10.3109/09687688.2010.507788

[5] O. Albrecht, D. S. Johnston, C. Villaverde and D. Chapman, "Stable Biomembrane Surfaces Formed by Phospholipid Polymers," Biochim Biophys Acta, Vol. 687, No. 2, 1982, pp. 165-169. doi:10.1016/0005-2736(82)90542-9

[6] R. Puri and A. R. Blumenthal, "Polymeric Lipid Assemblies as Novel Theranostic Tools," Accounts of Chemical Research, Vol. 44, No. 10, 2011, pp. 1071-1079. doi:10.1021/ar2001843

[7] M. Gou, G. Guo, J. Zhang, K. Men, J. Song, F. Luo, X. Zhao, Z. Qian and Y. Wei, "Time-Temperature Chromatic Sensor Based on Polydiacetylene (PDA) Vesicle and Amphiphilic Copolymer," Sensors and Actuators B: Chemical, Vol. 150, No. 1, 2010, pp. 406-411. doi:10.1016/j.snb.2010.06.041

[8] S. Alonso-Romanowski, N. S. Chiaramoni, V. S. Lioy, R. A. Gargini, L. I. Viera and M. C. Taira, "Characterization of Diacetylenic Liposomes as Carriers for Oral Vac- cines," Chemistry and Physics of Lipids, Vol. 122, No. 1-2, 2003, pp. 191-203. doi:10.1016/S0009-3084(02)00190-1

[9] G. Ravindran and W. L. Bryden, "Tryptophan Determination in Proteins and Feedstuffs by Ion Exchange Chromatography," Food Chemistry, Vol. 89, No. 2, 2005, pp. 309-314. doi:10.1016/j.foodchem.2004.05.035

[10] P. Nagaraja, H. S. Yathirajan and R. A. Vasantha, "Highly Sensitive Reaction of Tryptophan with $p$-Phenylenediamine," Analytical Biochemistry, Vol. 312, No. 2, 2003, pp. 157-161.

[11] G. W. Gokei, "Indole, the Aromatic Element of Tryptophan, as a Pi-Donor and Amphiphilic Headgroup," International Congress Series, Vol. 1304, 2007, pp. 1-14. doi:10.1016/j.ics.2007.07.038

[12] C. E. B. Esbjorner, B. Caesar, P. Albinsson, B. Lincoln and B. Norden, "Tryptophan Orientation in Model Lipid Membranes," Biochemical and Biophysical Research Communications, Vol. 361, No. 3, 2007, pp. 645-650. doi:10.1016/j.bbrc.2007.07.135

[13] A. V. Popova and D. K. Hincha, "Specific inTeractions of Tryptophan with Phosphatidylcholine and Digalacto- syldiacylglycerol in Pure and Mixed Bilayers in the Dry and Hydrated State," Chemistry and Physics of Lipids, Vol. 132, No. 2, 2004, pp. 171-184. doi:10.1016/j.chemphyslip.2004.06.003

[14] C. F. Temprana, E. L. Duarte, M. C. Taira, M. T. Lamy and S. del Valle Alonso, "Structural Characterization of Photopolymerizable Binary Liposomes Containing Diacetylenic and Saturated Phospholipids," Langmuir, Vol. 26, No. 12, 2010, pp. 10084-10092. doi:10.1021/la100214v

[15] R. J. Fernstrom and R. J. Wurtman, "Brain Serotonin Content: Physiological Dependence on Plasma Tryptophan Levels," Science, Vol. 173, No. 3992, 1971, pp. 149-152. doi:10.1126/science.173.3992.149

[16] R. J. Schaechter and R. J. Wurtman, "Tryptophan Availability Modulates Serotonin Release from Rat Hypothalamic Slices," Journal of Neurochemistry, Vol. 53, No. 6, 1989, pp. 1925-1933.

[17] F. Wurtman, E. Hefti and E. Melamed, "Precursor Control of Neurotransmitter Synthesis," Pharmacological Reviews, Vol. 32, No. 4, 1980, pp. 315-335.

[18] R. J. Wurtman and J. J. Wurtman, "Serotoninergic Mechanisms and Obesity," The Journal of Nutritional Biochemistry, Vol. 9, No. 9, 1998, pp. 511-515. doi:10.1016/S0955-2863(98)00029-1

[19] A. D. Bangham, "Model Membranes," Chemistry and Physics of Lipids, Vol. 8, No. 4, 1972, pp. 386-392. doi:10.1016/0009-3084(72)90069-2

[20] N. S. Chiaramoni, L. C. Baccarini, M. C. Taira and S. D. V. Alonso, "Liposome/DNA Systems: Correlation between Hydrophobicity and DNA Conformational Changes," Journal of Biological Physics, Vol. 34, No. 1-2, 2008, pp. 179-188. doi:10.1007/s10867-008-9103-2

[21] N. S. Chiaramoni, L. Speroni, M. C. Taira and S. D. V. Alonso, "Liposome/DNA Systems: Correlation between Association, Hydrophobicity and Cell Viability," Bio- 
technology Letters, Vol. 29, No. 11, 2007, pp. 1637-1644. doi:10.1007/s10529-007-9454-y

[22] M. M. Fabani, R. A. Gargini, M. C. Taira, R. Iacono, S. Iacono and S. Alonso-Romanowski, "Study of in Vitro Stability of Liposomes and in Vivo Antibody Response to Antigen Associated with Liposomes Containing GM1 after Oral and Subcutaneous Immunization," Journal of Liposome Research, Vol. 12, No. 1-2, 2002, pp. 13-27. doi:10.1081/LPR-120004772

[23] J. Gasparri, L. Speroni, N. S. Chiaramoni and S. del V. Alonso, "Relationship between the Adjuvant and Cytotoxic Effects of the Positive Charges and Polymerization in Liposomes," Journal of Liposome Research, Vol. 21, No. 2, 2011, pp. 124-133. doi:10.3109/08982104.2010.491073

[24] P. I. Lelkes and I. R. Miller, "Perturbations of Membrane Structure by Optical Probes: I. Location and Structural Sensitivity of Merocyanine 540 Bound to Phospholipid Membranes," The Journal of Membrane Biology, Vol. 52, No. 1, 1980, pp. 1-15. doi:10.1007/BF01869001

[25] J. Fogh, J. M. Fogh, J. Fau and T. Orfeo, "One Hundred and Twenty-Seven Cultured Human Tumor Cell Lines Producing Tumors in Nude Mice," Journal of the National Cancer Institute, Vol. 59, No. 1, 1997, pp. 221226.

[26] M. P. Desai, E. Labhasetwar, R. J. Walter, G. L. Levy and G. L. Amidon, "The Mechanism of Uptake of Biodegradable Microparticles in Caco-2 Cells Is Size Dependent," Pharmaceutical Research, Vol. 14, No. 11, 1997, pp. 1568-1573.

[27] T. Mosmann, "Rapid Colorimetric Assay for Cellular Growth and Survival: Application to Proliferation and Cytotoxicity Assays," Journal of Immunological Methods, Vol. 16, No. 1-2, 1983, pp. 55-63. doi:10.1016/0022-1759(83)90303-4

[28] M. C. Taira, N. S. Chiaramoni, K. M. Pecuch and S. Alonso-Romanowski, "Stability of Liposomal Formulations in Physiological Conditions for Oral Drug Delivery," Drug Delivery, Vol. 11, No. 2, 2004, pp. 123-128. doi:10.1080/10717540490280769

[29] S. Johnston, A. Sanghera, D. Manjon-Rubio and D. Chapman, "The Formation of Polymeric Model Biomembranes from Diacetylenic Fatty Acids and Phospholipids," Biochimica et Biophysica Acta, Vol. 602, No. 1, 1980, pp. 213-216.

[30] R. Ahl, J. Price, B. P. Smuda, A. Gaber and A. Singh, "Insertion of Bacteriorhodopsin into Polymerized Diacetylenic Phosphatidylcholine Bilayers," Biochimica et Biophysica Acta, Vol. 1028, No. 2, 1990, pp. 141-153. doi:10.1016/0005-2736(90)90148-H

[31] H. Norman and H. Nymeyer, "Indole Localization in Lipid Membranes Revealed by Molecular Simulation,"
Biophysical Journal, Vol. 91, No. 6, 2006, pp. 2046-2054. doi:10.1529/biophysj.105.080275

[32] I. Stanish and A. Singh, "Highly Stable Vesicles Composed of a New Chain-Terminus Acetylenic Photopolymeric Phospholipid," Chemistry and Physics of Lipids, Vol. 112, No. 2, 2001, pp. 99-108. doi:10.1016/S0009-3084(01)00173-6

[33] I. Fournier, J. Barwicz, M. Auger and P. Tancrède, “The Chain Conformational Order of Ergosterol- or Cholesterol-Containing DPPC Bilayers as Modulated by Amphotericin B: A FTIR Study," Chemistry and Physics of Lipids, Vol. 151, No. 1, 2008, pp. 41-50. doi:10.1016/j.chemphyslip.2007.09.006

[34] D. A. Mannock, R. N. A. H. Lewis and R. N. McElhaney, "A Calorimetric and Spectroscopic Comparison of the Effects of Ergosterol and Cholesterol on the Thermotropic Phase Behavior and Organization of Dipalmitoylphosphatidylcholine Bilayer Membranes," Biochimica et Biophysica Acta (BBA)-Biomembranes, Vol. 1798, No. 3, 2010, pp. 376-388.

[35] R. N. A. H. Lewis and R. N. McElhaney, "The Structure and Organization of Phospholipid Bilayers as Revealed by Infrared Spectroscopy," Chemistry and Physics of Lipids, Vol. 96, No. 1-2, 1998, pp. 9-21. doi:10.1016/S0009-3084(98)00077-2

[36] P. L. Ahl and W. R. Perkins, "Interdigitation-Fusion Liposomes," In: D. Nejat, Ed., Methods in Enzymology, Vol. 367, Academic Press, Cambridge, 2003, pp. 80-98.

[37] A. M. A. Elhissi, M. A. A. O'Neill, S. A. Roberts and K. M. G. Taylor, "A Calorimetric Study of Dimyristoylphosphatidylcholine Phase Transitions and Steroid-Liposome Interactions for Liposomes Prepared by Thin Film and Proliposome Methods," International Journal of Pharmaceutics, Vol. 320, No. 2, 2006, pp. 124-130.

[38] K. Takeda, H. Okuno, T. Hata, M. Nishimoto, H. Matsuki and S. Kaneshina, "Interdigitation and Vesicle-to-Micelle Transformation Induced by a Local Anesthetic Tetracaine in Phospholipids Bilayers," Colloids and Surfaces B: Biointerfaces, Vol. 72, No. 1, 2009, pp. 135-140.

[39] P. R. Griffiths, "The Handbook of Infrared and Raman Characteristic Frequencies of Organic Molecules," Vibrational Spectroscopy, Vol. 4, No. 1, 1992, p. 121. doi:10.1016/0924-2031(92)87021-7

[40] O. Takikawa, "Biochemical and Medical Aspects of the Indoleamine 2,3-Dioxygenase-Initiated 1-Tryptophan Metabolism," Biochemical and Biophysical Research Communications, Vol. 338, No. 1, 2005, pp. 12-19. doi:10.1016/j.bbrc.2005.09.032

[41] N. Le Floc'h and B. Seve, "Biological Roles of Tryptophan and Its Metabolism: Potential Implications for Pig Feeding," Livestock Science, Vol. 112, No. 1, 2007, pp. 23-32. doi:10.1016/j.livsci.2007.07.002 